\title{
Cryocompression Therapy
}

National Cancer Institute

\section{Source}

National Cancer Institute. Cryocompression Therapy. NCI Thesaurus. Code $C 157773$.

Therapy that combines compression garments or dressings with cooling of the treated area. 